\title{
Chaotic Model for Lévy Walks in Swarming Bacteria
}

\author{
Gil Ariel, ${ }^{1}$ Avraham Be'er, ${ }^{2}$ and Andy Reynolds ${ }^{3, *}$ \\ ${ }^{1}$ Department of Mathematics, Bar-Ilan University, Ramat Gan 52000, Israel \\ ${ }^{2}$ Zuckerberg Institute for Water Research, The Jacob Blaustein Institutes for Desert Research, \\ Ben-Gurion University of the Negev, Sede Boqer Campus 84990, Midreshet Ben-Gurion, Israel \\ ${ }^{3}$ Rothamsted Research, Harpenden, Herefordshire AL5 2JQ, United Kingdom
}

(Received 16 January 2017; revised manuscript received 8 March 2017; published 2 June 2017)

We describe a new mechanism for Lévy walks, explaining the recently observed superdiffusion of swarming bacteria. The model hinges on several key physical properties of bacteria, such as an elongated cell shape, self-propulsion, and a collectively generated regular vortexlike flow. In particular, chaos and Lévy walking are a consequence of group dynamics. The model explains how cells can fine-tune the geometric properties of their trajectories. Experiments confirm the spectrum of these patterns in fluorescently labeled swarming Bacillus subtilis.

DOI: 10.1103/PhysRevLett.118.228102

Bacterial swarming is a mode of motion in which flagellated cells move collectively over surfaces, producing coherent swirling flows [1-6]; see Figs. 1(a) and 1(b). The physical principles underlying bacterial swarming, as well as the statistical properties of its dynamics, have been extensively analyzed, both experimentally and in models $[2,7,8]$. In particular, it has been shown that the continuous motion of individual bacteria inside the swarm is undirected and may be independent of the chemotactic signaling systems [3,9]. Recently, by analyzing trajectories of fluorescently labeled cells moving within a dense swarm, Ariel et al. [10] showed that the erratic movements of individual bacteria closely resemble Lévy walks (LWs) (Fig. 1). This is in contrast to sparsely swimming bacteria that move by a process called run and tumble, in which cells move for relatively short times (typically up to a few seconds) in straight trajectories (runs) interspersed by rapid reorientations (tumbles) [7]. Thus, Lévy walking appears to be related to the biological or physical mechanisms underlying the collective swarming phenomenon in bacteria.

The key to understanding and predicting many phenomena lies with the identification of an underlying generative mechanism [11]. Many putative mechanisms have been identified for the generation of LWs by noninteracting individuals $[12,13]$. These include external mechanisms such as trail following with obstacles or random odor trails, power laws that are inherent to the movement mechanisms (e.g., the odometer in bees or slick-slip locomotion in cells), and chaos on the neurological level. Few models explain LWs in weakly interacting individuals [14]. It has been suggested that isolated bacteria may follow a LW pattern [15], but this has not been observed experimentally. Swarming bacteria are, however, highly dense, and cell-cell interactions dominate the dynamics. Hence, the identification of a mechanism explaining LWs in swarming bacteria presents a completely new scenario. Moreover, it may be applicable to other strongly interacting self-propelled particles or organisms.

In this Letter, we show the emergence of chaotic dynamics in cells that are advected by the collective flow of the swarm. The emergence of LWs in inanimate particles moving along a chaotic [16] or time-dependent periodic fields [17] is well known. However, these models do not
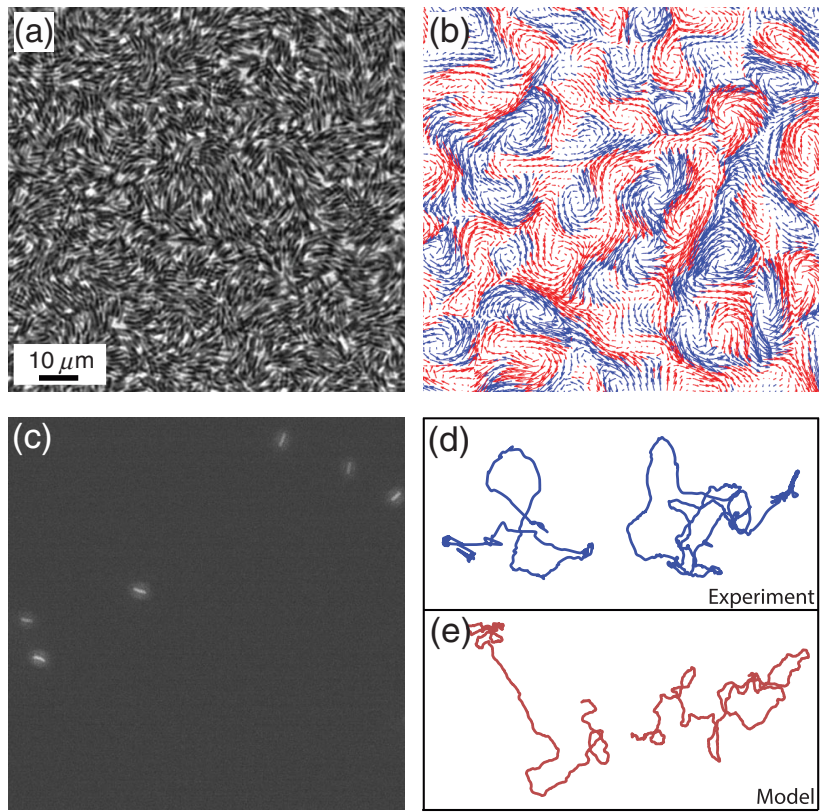

FIG. 1. Dynamics of individual bacteria within a dense swarm. (a) Optical microscopy image. (b) The collective flow of the swarm reveals a swirling dynamical flow pattern. (c) A low density of fluorescently labeled cells observed within the dense swarm. (d) Example trajectories obtained in experiments show superdiffusive behavior and fit a Weierstrassian Lévy walk model well. (e) Simulated trajectories show a Lévy walk pattern generated by chaotic dynamics. 
take into account several physical properties that are known to be fundamental for the bacterial ability to swarm effectively, particularly self-propulsion and an elongated shape [1-8]. In our model, the flow field is not chaotic itself (it may even be periodic). Instead, chaotic motion originates from the ability of bacteria to actively shift between flow lines using their self-generated thrust. Loosely speaking, chaos results because the periodicity of orientation is generally not rationally related to the periodicity of the flow. In our model, the governing equations form a threedimensional system or autonomous ordinary differential equations, which is the lowest dimension for an unforced continuous dynamical system to exhibit chaotic motion. It presents a new model for the appearance of chaos and LWs in such systems.

A key results of our model is that the emergence of a LW and its statistics sensitively depends on individual cell parameters such as the cell shape and the thrust exerted by the flagella. This observation has significant biological implications as it shows that the geometrical properties of cell trajectories can be fine-tuned by selection pressures. It has been postulated that LWs can be advantageous when foraging probabilistically without any knowledge of target locations (also known as the Lévy flight foraging hypothesis) $[12,18]$. Indeed, we identify some ways in which LWs could be advantageous for swarming bacteria.

The physical properties of swarming, or collectively swimming bacteria at high density, have been extensively studied in experiments $[1,2,4-6,8]$. Based on these studies, several models have been suggested to explain the seemingly erratic swirling flow patterns observed in systems of selfpropelled rodlike particles and, in particular, bacteria [5,19]. In order to highlight the single cell dynamics, we take a simplified approach and suggest a model describing the motion of a single cell within the effective flow of the swarm. This approach is motivated by sedimentation models which were shown to have chaotic dynamics [20]. In particular, we draw inspiration from Mallier and Maxey [21], who studied the gravitational settling of spheroidal particles in an incompressible, steady Stokes flow.

Our model is based on the following established assumptions regarding the physical properties of swarming bacteria $[1,2,4-6,8]$. (a) Bacteria are elongated rodlike objects. (b) Cells are self-propelled, pushing in the direction of the axis of symmetry. Bacterial colonies are characterized by low Reynolds numbers (typically $10^{-4}-10^{-3}$ ), and the dynamics of the particles is well approximated by the Stokes flow regime. Because of the highly viscous medium, pushing rapidly relaxes to a terminal speed $V$. (c) The swarm creates an effective flow approximated by an array of vortices. (d) Because of their small cell size, the stress tensor across a cell is approximately uniform. The effect of a single cell on the collective flow (which may be generated by millions of bacteria) is negligible. To be precise, let $(x(t), y(t))$ denote the position of the cell at time $t$ and let $\hat{m}(t)=(\cos \theta(t), \sin \theta(t))^{T}$ denote a unit vector pointing in the direction of the front of the cell. For simplicity, we initially assume a periodic flow pattern with streamline function $\psi(x, y)=\pi^{-1} \sin \pi x \cos \pi x$. This is clearly a major simplification of the complex bacterial flow $[1,6]$. Nonetheless, we show that it is sufficient for explaining the emergence of LWs in these systems and other essential properties of the dynamics. More complicated stream functions, including slowly varying ones, are studied in Sec. I of the Supplemental Material (SM) [22]. Then, the dynamics of a test cell (or particle) is given by

$$
\begin{aligned}
\left(\begin{array}{c}
\dot{x} \\
\dot{y}
\end{array}\right) & =\left(\begin{array}{c}
\sin \pi x \cos \pi y \\
-\cos \pi x \sin \pi y
\end{array}\right)+V\left(\begin{array}{c}
\cos \theta \\
\sin \theta
\end{array}\right) \\
\dot{\theta} & =\pi \sin \pi x \sin \pi y-2 D \pi \cos \pi x \cos \pi y \cos \theta \sin \theta .
\end{aligned}
$$

The first term in the equation for the velocity $(\dot{x}, \dot{y})^{T}$ describes advection due to the flow $u(x, y)=$ $(\partial \psi / \partial y,-\partial \psi / \partial x)^{T}$. Here, $(\cdots)^{T}$ denotes the transpose of a vector. The second term describes the self-propulsion force acting in the direction $\hat{m}$, resulting in a terminal speed $V$. The first term in the equation for the radial velocity $\dot{\theta}$ describes rotation due to the vorticity of the flow, and the second term is rotation due to shear (also known as Jeffery's equation). The constant $D$ depends on the shape of the particle. For a prolate spheroid $D=\left(\lambda^{2}-1\right) /\left(\lambda^{2}+1\right)$, where $\lambda$ is the aspect ratio of the cell (approximately 5 for $B$. subtilis). With the stream function described above, each vortex is a $1 \times 1$ square, and a passive sphere at a vortex center rotates once every unit time. This sets the temporal and spatial scales. See Sec. I of the SM [22] for a detailed derivation and generalizations to more complicated and fluctuating flow fields. Figure 2(a) depicts the flow field and a sample trajectory. See also Fig. S1 of the SM [22].

Simulated trajectories with biologically realistic parameters, $\lambda=5$ (a $5: 1$ aspect ratio) and $V=1 / 2$ (in experiments, the speed of a single bacterium swimming individually is about half the average speed inside a swarm $[2,3,7,8,10]$ ), are consistent with LW movement patterns. Later, we show that LWs occur for a wide range of aspect ratios and speeds. Figure 2(b) shows the mean-squared displacement (MSD), which scales as $t^{\alpha}$, exhibiting superdiffusion with an exponent of approximately $\alpha=1.65$. Superdiffusion is not observed either with inert particles $(V=0)$, in which case trajectories are periodic, or with spheres $(\lambda=1$ or $D=0)$, for which our model predicts normal diffusion (Fig. S2 of the SM [22]). Indeed, tracking experiments confirm that fluorescently labeled immotile bacteria (i.e., non-self-propelled bacteria with $V=0$ ) embedded within a motile swarm undergo normal diffusion. See Fig. S3 and Secs. IV-VI of the SM [22]. The absence of superdiffusion in these cases shows that LWs in swarming bacteria and in inert spherical 

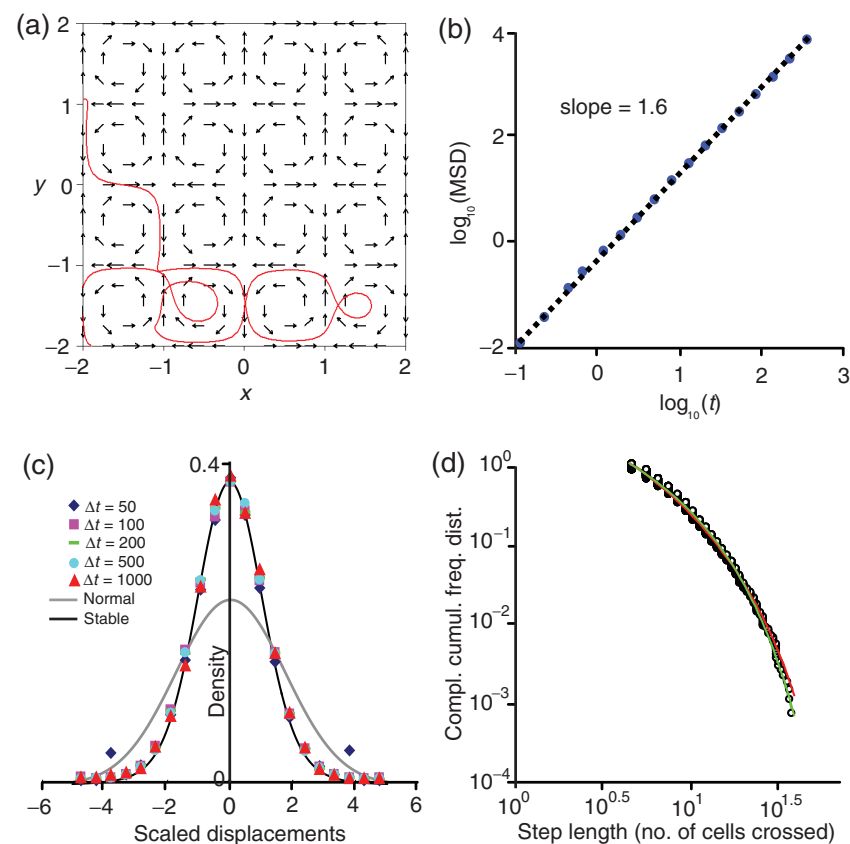

FIG. 2. Simulations with biologically realistic parameters. (a) A sample trajectory of a nonspherical particle (red) in a doubleperiodic flow (the black arrows). (b) The mean-squared displacement shows superdiffusive behavior with an exponent of 1.65. (c) The density of displacements $p(\Delta t, \Delta x)$, scaled by a factor of $\Delta t^{1 / \beta}(\beta=1.22)$, fits a Lévy stable distribution with exponent $3-\beta$ well. (d) The complement of the cumulative frequency distribution for the observed step lengths in simulated trajectories (०), the best-fit WRW (green), and the best-fit Lévy walk model (red). The WRW fit implies a power-law exponent $\mu=1.45$.

particles within rotating flows have fundamentally different origins, as the latter has been attributed to "chaos within the flow" [28,29]. See Sec. II and Fig. S4 of the SM [22] for a discussion on model parameters and their effects on the dynamics.

In addition, Fig. 2(c) shows the density of displacements $p(\Delta t, \Delta x)$, scaled by a factor of $\Delta t^{1 / \beta}$. With $\beta=1.22$, displacements with different values of $\Delta t$ approximately collapse on a master curve (compare this with the expected $\beta=3-\alpha$ for LWs [30]) that fits a Lévy stable distribution with exponent $3-\beta$ well. Figure 2(d) shows the complement of the cumulative frequency distribution for the observed step lengths in 100 simulated trajectories together with the best-fit Weierstrassian random walk (WRW) and other LW models (Sec. III of the SM [22]).

Weierstrassian random walks are one of the simplest random walks, which do not satisfy the central limit theorem and, as such, have come to epitomize scale invariance [31]. They are characterized by a hierarchical step-length distribution with density $[32,33]$

$$
p(l)=\frac{q-1}{q} \sum_{j=0}^{\infty} q^{-j} b^{-(j+1)} \exp \left(-l / b^{j+1}\right) .
$$

WRW satisfy a self-similar scaling form such that orderof-magnitude longer steps occur an order of magnitude less often; i.e., a step drawn from an exponential distribution with mean $b^{j+1}$ is $q$ times more likely than is a step drawn from an exponential with the next longest mean. Consequently, a walker will typically make a cluster of $q$ steps with mean $b$ before making a step of length $b^{2}$, and so initiating a new cluster. About $q$ such clusters separated by a distance of order $b$ are formed before a step of length $b^{2}$ is made, and so on. Eventually, a hierarchy of clusters within clusters is formed. This is the hallmark of a Lévy walk. It is readily shown that the step-length distribution, Eq. (2), has infinite variance when $b^{2}>q$ and corresponds to a Lévy walk with exponent $\mu=\beta+1=1+\ln q / \ln b$. By comparison, conventional LWs draw step lengths from probability distributions with heavy power-law tails (typically a power law with exponent $\mu=3-\alpha$ ). The hierarchical structure of Weierstrassian Lévy walks is intrinsically related to the self-similar topological and dynamical properties of the orbits $[34,35]$.

It is now generally accepted that power spectra with exponential frequency dependency are a unique intrinsic and observable signature of systems exhibiting deterministic chaos [36,37]. Such spectra have been observed in theoretical models of chaos and in experimental studies of fluid flows and confined plasmas [29,38]. In accordance with these observations, numerical solutions of Eq. (2) confirm that trajectories are indeed chaotic. For example, Fig. 3(a) shows that the power spectrum is exponential. Figure 3(b) shows that the largest Lyapunov exponent is approximately 1.2, where a positive value indicates chaos. See Sec. VII of the SM [22] for details. Finally, Poincaré return maps [Figs. 3(c) and 3(d)] show a complex structure, as expected for chaotic trajectories. These observations are robust and persist with slowly varying flow fields as well (see Sec. I of the SM [22]). In order to confirm the applicability of our model to swarming bacteria, we analyzed a data set of 58 trajectories of fluorescently labeled B. subtilis cells embedded within a high-density swarming colony (See Secs. IV and V of the SM [22] and Ref. [10]). We find that movement patterns closely resemble a five-tier WRW with Lévy exponent 1.75 [Fig. 4(a)]. The Akaike weight for the WRW is 1.00. Moreover, the power spectrum [Fig. 4(b)] has exponential frequency dependency, as expected for chaotic trajectories $[35,36]$.

Movement patterns resembling LWs have been identified in a wide variety of organisms, from cells to humans [39]. Ariel et al. [10] were the first to report on LWs within a group of strongly interacting organisms: a bacterial swarm. The results of our analyses suggest that Lévy walk movement patterns occurring within bacterial swarms may have arisen freely as a mathematical consequence of the collective dynamics. Their occurrence can be attributed to the presence of generic properties of chaos (long-lived orbits in phase space) $[34,35]$; properties which would not persist in 

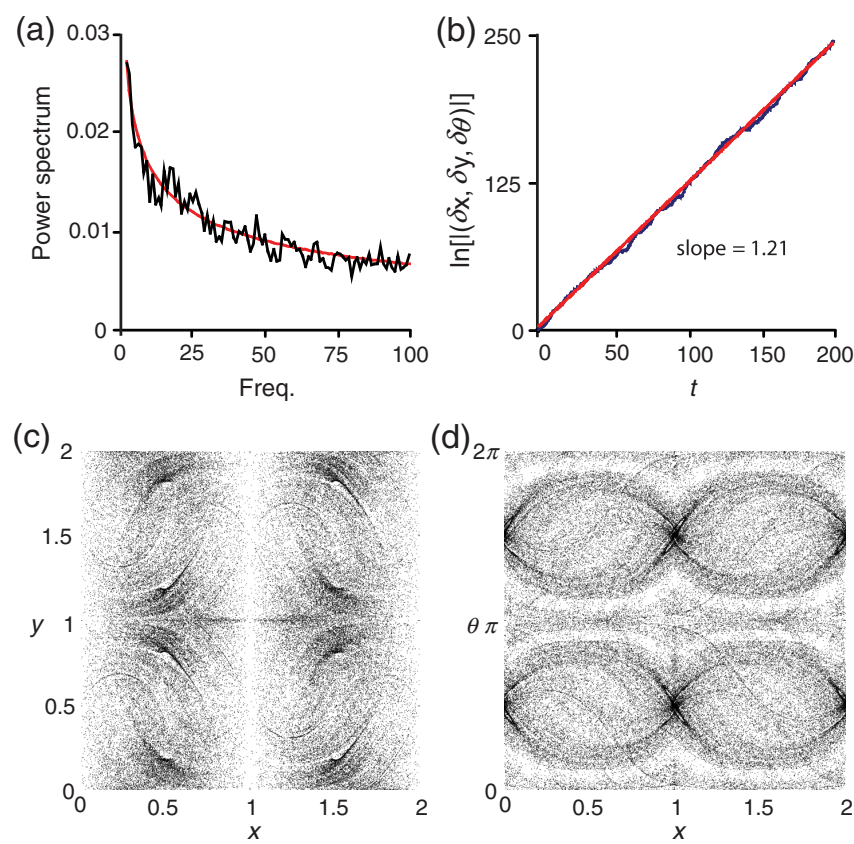

FIG. 3. Simulated trajectories show chaos. (a) Ensemble average power spectrum (the black line) and a best-fit stretched exponential (the red line). (b) A numerical approximation of the Lyapunov exponent indicates chaotic dynamics. (c),(d) Poincaré return maps showing successive recordings of a particle position $(x, y) \bmod 2$ at (c) $\theta=0$ and (d) $y=0$.

the presence of significant disorder or in the presence of significant thermal fluctuations. This dispenses with the need to understand how such complex movement patterns could have evolved from simpler, finite-scale processes. In this regard, Lévy walks in bacterial swarms are no different than any of the other examples of biological LWs whose occurrences can be attributed to seemingly innocuous, undirected processes [13]. Nonetheless, Lévy walks in bacterial swarms, as well as other biological and ecological systems, could be advantageous, and in this case there could be a selection for maintaining them [13]. In this regard, it is noteworthy that, in contrast to most other generative mechanisms for biological Lévy walks [13], chaotically generated LWs are plastic (i.e., they can be tuned, for example, by adjusting the speed of selfpropulsion), which is a prerequisite for the Lévy flight foraging hypothesis [18]. For example, Fig. 5 depicts the dependency of the Lévy exponent $\mu$ on the cell aspect ratio for several biologically realistic values of pushing speeds $V$. Interestingly, we find that the lowest Lévy exponent (the most superdiffusive process) is obtained at an aspect ratio of around 5, which is approximately the cell aspect ratio observed in many wild-type swarming bacterial species. This finding suggests a novel selection mechanism that regulates the observed aspect ratio of cells as a consequence of the physics underlying bacterial swarming.

Whether or not the Lévy walk movements are advantageous for swarming bacteria remains an open question, one
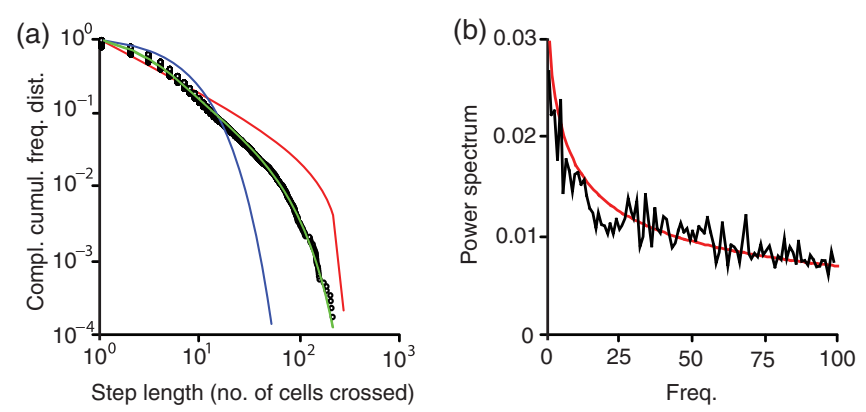

FIG. 4. Experimental results with swarming B. subtilis. (a) The complement of the cumulative frequency distribution for the observed step lengths in individual cells $\left(^{\circ}\right)$ and the best-fit WRW (Weierstrassian Lévy walk) (the green line), Lévy (power-law) walk model (the red line), and Brownian (exponential) walk (the blue line). (b) Ensemble average power spectrum (the black line) and the best-fit stretched exponential (the red line).

which we begin to explore in Sec. VIII of the SM [22]. Our new model for the emergence of LWs as an interplay between the dynamics of an individual and that of the collective suggests that such Lévy walks may not be specific to bacterial swarms, as they may also occur in flocks and swarms of higher organisms, as exemplified perhaps in fish schools [40]. Much early research into collective animal behavior was concerned with the broad question of why animals form aggregations [41]. This research led to the realization that animals within aggregations may benefit from social interactions [42], for example, by increased protection from predators [43], increased locomotion efficiency, and enhanced foraging success - the many eyes hypothesis [44]. Our new results suggest a new mechanism favoring group dynamics by way of fundamentally changing the geometrical properties of individual trajectories. This warrants further investigation,

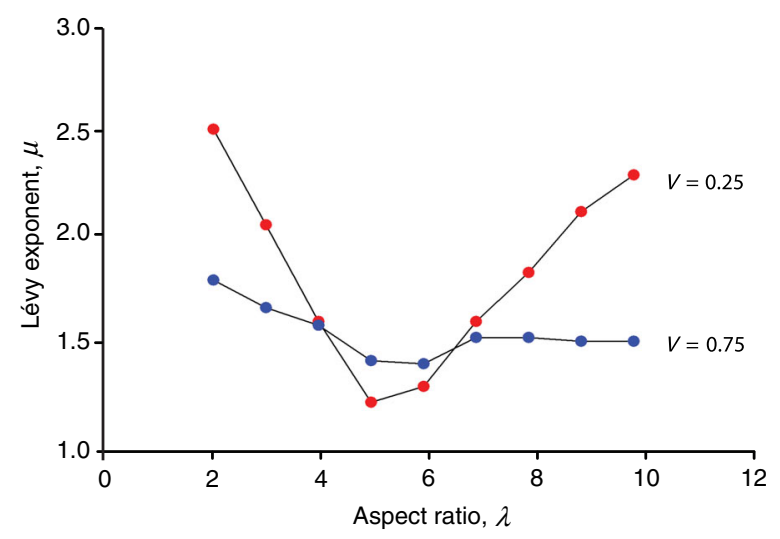

FIG. 5. The dependence of the Lévy exponent $\mu$ on the aspect ratio $\lambda$ for different speeds $V$. For $\mu>3$, steps have a finite variance, implying normal diffusion. For a range of physically realistic speeds, the lowest Lévy exponent (the most superdiffusive) is obtained at an aspect ratio of around 5, similar to swarming Bacillus subtilis. 
opening up new perspectives on the physical mechanisms underlying Lévy walks as models of movement patterns and their evolutionary origins.

We thank Ed Ott for discussions. G. A. acknowledges partial support from the National Science Foundation Research Network on kinetic equations (KI-Net) under Grants No. 1107444 and No. 1107465; A. B. and G. A. acknowledge partial support from The Israel Science Foundation (Grant No. 373/16). Rothamsted Research receives grant aided support from the Biotechnology and Biological Sciences Research Council.

* Corresponding author.

andy.reynolds@rothamsted.ac.uk

[1] R. M. Harshey, Annu. Rev. Microbiol. 57, 249 (2003); D. B. Kearns and R. Losick, Mol. Microbiol. 49, 581 (2004); L. H. Cisneros, R. Cortez, C. Dombrowski, R. E. Goldstein, and J. O. Kessler, Exp. Fluids 43, 737 (2007); A. Sokolov, I. S. Aranson, J. O. Kessler, and R. E. Goldstein, Phys. Rev. Lett. 98, 158102 (2007); C. W. Wolgemuth, Biophys. J. 95, 1564 (2008); J. P Hernandez-Ortiz, P. T Underhill, and M. D Graham, J. Phys. Condens. Matter 21, 204107 (2009); A. Sokolov and I. S. Aranson, Phys. Rev. Lett. 103, 148101 (2009); H. P. Zhang, A. Be'er, R. S. Smith, E.-L. Florin, and H. L. Swinney, Europhys. Lett. 87, 48011 (2009).

[2] N. C. Darnton, L. Turner, S. Rojevsky, and H. C. Berg, Biophys. J. 98, 2082 (2010).

[3] D. B. Kearns, Nat. Rev. Microbiol. 8, 634 (2010).

[4] H. P. Zhang, A. Be'er, E.- L. Florin, and H. L. Swinney, Proc. Natl. Acad. Sci. U.S.A. 107, 13626 (2010); X. Chen, X. Dong, A. Be'er, H. L. Swinney, and H. P. Zhang, Phys. Rev. Lett. 108, 148101 (2012).

[5] H. H. Wensink, J. Dunkel, S. Heidenreich, K. Drescher, R. E. Goldstein, H. Lowen, and J. M. Yeomans, Proc. Natl. Acad. Sci. U.S.A. 109, 14308 (2012).

[6] J. Dunkel, S. Heidenreich, K. Drescher, H. H. Wensink, M. Bär, and R. E. Goldstein, Phys. Rev. Lett. 110, 228102 (2013); A. Rabani, G. Ariel, and A. Be'er, PLoS One 8, e83760 (2013); S. Benisty, E. Ben-Jacob, G. Ariel, and A. Be'er, Phys. Rev. Lett. 114, 018105 (2015).

[7] H. C. Berg, E. Coli in Motion (Springer, New York, 2004).

[8] A. Sokolov and I. S. Aranson, Phys. Rev. Lett. 109, 248109 (2012).

[9] J. D. Partridge and R. M. Harshey, J. Bacteriol. 195, 909 (2013).

[10] G. Ariel, A. Rabani, S. Benisty, J. D. Partridge, R. M. Harshey, and A. Be'er, Nat. Commun. 6, e8396 (2015).

[11] S. A. Levin, Ecology 73, e1943 (1992); V. Zaburdeav, S. Denisov, and J. Klafter, Rev. Mod. Phys. 87, 483 (2015).

[12] G. M. Viswanathan et al., The Physics of Foraging (Cambridge University Press, Cambridge, England, 2011).

[13] A. M. Reynolds, Phys. Life Rev. 14, 59 (2015).

[14] A. M. Reynolds and S. A. H. Geritz, Physica (Amsterdam) 424A, 317 (2015); A. M. Reynolds and N. T. Ouellette, Sci. Rep. 6, 30515 (2016).
[15] E. Korobkova, T. Emonet, J. M. G. Vilar, T. S. Shimizu, and P. Cluzel, Nature (London) 428, 574 (2004); Y. Tu and G. Grinstein, Phys. Rev. Lett. 94, 208101 (2005).

[16] T. Geisel, J. Nierwetberg, and A. Zacherl, Phys. Rev. Lett. 54, 616 (1985); A. Zacherl, T. Geisel, J. Nierwetberg, and G. Radons, Phys. Lett. 114A, 317 (1986); T. Geisel, A. Zacherl, and G. Radons, Phys. Rev. Lett. 59, 2503 (1987).

[17] I. S. Aranson, M. I. Rabinovich, and L.Sh. Tsimring, Phys. Lett. A 151, 523 (1990); I. S. Aranson, A. B. Ezersky, M. I. Rabinovich, and L.S. Tsimring, Phys. Lett. A 153, 211 (1991); O. N. Mesquita, S. Kane, and J. P. Gollub, Phys. Rev. A 45, 3700 (1992); T. H. Solomon, A. T. Lee, and M. A. Fogleman, Physica (Amsterdam) 157D, 40 (2001).

[18] G. M. Viswanathan, E. P. Raposo, and M. G. E. da Luz, Phys. Life Rev. 5, 133 (2008).

[19] R. A. Simha and S. Ramaswamy, Phys. Rev. Lett. 89, 058101 (2002); F. Peruani, A. Deutsch, and M. Bär, Phys. Rev. E 74, 030904 (2006); I. S. Aranson, A. Sokolov, J. O. Kessler, and R. E. Goldstein, Phys. Rev. E 75, 040901(R) (2007); D. Saintillan and M. J. Shelley, Phys. Rev. Lett. 99, 058102 (2007); N. Sambelashvili, A. W. C. Lau, and D. Cai, Phys. Lett. A 360, 507 (2007); S. Sankararaman and S. Ramaswamy, Phys. Rev. Lett. 102, 118107 (2009); S. D. Ryan, B. M. Haines, L. Berlyand, F. Ziebert, and I. S. Aranson, Phys. Rev. E 83, 050904(R) (2011); F. Peruani, J. Starruß, V. Jakovljevic, L. S. gaard-Andersen, A. Deutsch, and M. Bär, Phys. Rev. Lett. 108, 098102 (2012); S. D Ryan, A. Sokolov, L. Berlyand, and I. S Aranson, New J. Phys. 15, 105021 (2013); S. Weitz, A. Deutsch, and F. Peruani, Phys. Rev. E 92, 012322 (2015).

[20] L. Yu, C. Grebogi, and E. Ott, in Nonlinear Structure in Physical Systems, edited by L. Lam and H. C. Morris (Springer-Verlag, New York, 1990), p. 223.

[21] R. Mallier and M. Maxey, Phys. Fluids A 3, 1481 (1991).

[22] See Supplemental Material at http://link.aps.org/ supplemental/10.1103/PhysRevLett.118.228102 for generalizations of our model to more complicated stream functions, which includes Refs. [23-27].

[23] A. Clauset, C. R. Shalizi, and M. E. J. Newman, SIAM Rev. 51, 661 (2009).

[24] A. M. Edwards et al., Nature (London) 449, 1044 (2007).

[25] A. M Reynolds, F. Bartumeus, A. Kölzsch, and J. van de Koppel, Sci. Rep. 6, 23492 (2016).

[26] H. Hakli and H. Uuz, Appl. Soft Comput. 23, 333 (2014).

[27] H. G. Schuster and W. Just, Deterministic Chaos (Wiley, New York, 2005).

[28] T. H. Solomon, E. R. Weeks, and H. L. Swinney, Phys. Rev. Lett. 71, 3975 (1993).

[29] A. Brandstater and H. L. Swinney, Phys. Rev. A 35, 2207 (1987).

[30] J. Klafter, M. F. Schlesinger, and G. Zumofen, Phys. Today 49, 33 (1996).

[31] B. D. Hughes, M. F. Schlesinger, and E. W. Montroll, Proc. Natl. Acad. Sci. U.S.A. 78, 3287 (1981).

[32] Note that this form, which is more applicable to a moving organism, is different than the WRW defined in Ref. [31].

[33] A. M. Reynolds, Sci. Rep. 4, 4409 (2014).

[34] G. M. Zaslavsky, D. Stevens, and H. Weitzner, Phys. Rev. E 48, 1683 (1993). 
[35] G. M. Zaslavsky, Chaos 5, 653 (1995).

[36] U. Frisch and R. Morf, Phys. Rev. A 23, 2673 (1981).

[37] A. Libchaber, S. Fauve, and C. Laroche, Physica (Amsterdam) 7D, 73 (1983).

[38] M. R. Paul, M. C. Cross, P. F. Fischer, and H. S. Greenside, Phys. Rev. Lett. 87, 154501 (2001).

[39] A. M. Reynolds, A. D. Smith, R. Menzel, U. Greggers, D. R. Reynolds, and J. R. Riley, Ecology 88, 1955 (2007); D. W. Sims et al., Nature (London) 451, 1098 (2008); T. H. Harris et al., Nature (London) 486, 545 (2012); N. E. Humphries, H. Weimerskirch, and D. W. Sims, Methods Ecol. Evol. 4, 930 (2013); D. A. Raichlen, B. M. Wood, A. D. Gordon, A. Z. P. Mabulla, F. W. Marlowe, and H. Pontzer, Proc. Natl. Acad. Sci. U.S.A. 111, 728 (2014);
D. W. Sims, A. M. Reynolds, N. E. Humphries, E. J. Southall, V. J. Wearmouth, B. Metcalfe, and R. J. Twitchett, Proc. Natl. Acad. Sci. U.S.A. 111, 11073 (2014).

[40] H. Murakami, T. Niizato, T. Tomaru, Y. Nishiyama, and Y.-P. Gunji, Sci. Rep. 5, 10605 (2015).

[41] L.-A. Giraldeau and T. Caraco, Social Foraging Theory (Princeton University Press, Princeton, NJ, 2000); J. Krause and G. D. Ruxton, Living in Groups (Oxford University Press, Oxford, 2002).

[42] B. L. Partridge, T. Pitcher, J. M. Cullen, and J. Wilson, Behav. Ecol. Sociobiol. 6, 277 (1980).

[43] W. D. Hamilton, Theor. Popul. Biol. 31, 295 (1971).

[44] G. Turner and T. Pitcher, Am. Nat. 128, 228 (1986). 